\title{
PROBABILITY OF KNOTS IN A POLYMER RING
}

\author{
J.P.J. MICHELS \\ Van der Waals Laboratory, University of Amsterdam, P.O. Box 20216, 1000 HE Amsterdam, The Netherlands \\ and \\ F.W. WIEGEL \\ Department of Applied Physics, Twente University of Technology, \\ P.O. Box 217, 7500 AE Enschede, The Netherlands
}

Received 17 May 1982

\begin{abstract}
We generate equilibrium configurations of a ring polymer in an infinite space, or confined to the interior of a sphere. Using a new algorithm, the a priori probability for the occurrence of a knot is determined numerically. The results are compatible with power laws and scaling laws of striking simplicity.
\end{abstract}

One of the outstanding unsolved problems of the theory of macromolecular systems consists of classifying the topologically different classes of configurations of a single closed macromolecule. This problem was first recognized by Delbrück [1] and has subsequently been studied by several authors [2-14]. It is also related to the problem of the mutual entanglements between two macromolecules (links, cf. refs. [15-23]), as well as to certain topological problems which play a role in the Aharonov-Bohm effect [24-27]. In view of the almost unsurmountable difficulties in developing an analytical theory, there is a need for reliable numerical studies.

Numerical enumerations should have the following desirable properties: (a) Random walks have to be generated with the correct a priori probabilities, and in such a way that the ends are joined properly. (b) Given a macromolecular configuration, its topological properties should be determined in a mathematically unique way. (c) All numerical algorithms should be efficient enough to achieve adequate statistical accuracy. This numerical approach can properly be called

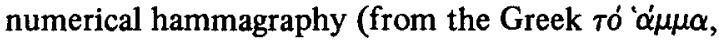
meaning the knot).

In their pioneering study (cf. appendix of ref. [3]) the Russian group generated random walks on a cubic lattice. Another approach was followed by des Cloizeaux and Mehta [5] who represented the chain by a gaussian random walk in continuous space. Strictly speaking, both approaches $[3,5]$ show a flaw in the way in which the end points of the chain are joined. This is accomplished by making the one-step distribution function depend on the position along the backbone of the macromolecule in such a way that the end points are forced to coincide. This violates the independence of the different steps in the random walk. This flaw was recognized and obviated by Chen [12] who made rings by dimerization of two free random walks. One of the aims of our work is to use an a priori closed ring model with repeating units of equal lengths.

Apart from the early work of Crippen [2] all authors use the Alexander polynomial $\Delta(t)$ to characterize the topological properties of a knot. We follow this by now established tradition. Yet it should be stressed that the fact that two knots have the same Alexander polynomial does not necessarily imply that they are topologically equivalent.

All previous authors note that few knots form in the short chains that they were able to study numerically. We have, therefore, made an effort to improve the efficiency of the algorithm in such a way that long chains with a high probability of knot formation 
can still be studied in large numbers.

Ring-shaped macromolecules are represented by sequences, each consisting of $N$ masspoints at positions $r_{1}, \ldots ., r_{N}$. Between any pair $(i, i+1)$ of nearest neighbours along the chain, we assume a harmonic force around a fixed distance $l$.

$\boldsymbol{F}_{i, i+1}=-k\left(\left|\boldsymbol{r}_{i+1}-\boldsymbol{r}_{i}\right|-l\right) \frac{\boldsymbol{r}_{i+1}-\boldsymbol{r}_{i}}{\left|\boldsymbol{r}_{i+1}-\boldsymbol{r}_{i}\right|}$.

Here $i=1, \ldots, N$ and $i+1=1$ if $i=N$. On mass point $i$ a random force acts, which models the heat motion of the surrounding solvent. Moreover, a frictional force, proportional to the velocity, acts on each mass point.

At time zero the molecule is assigned some initial configuration. The equations of motion for the $N$ mass points are solved numerically with methods commonly used in molecular dynamical simulations [28-30]. Once thermal equilibrium has been established, the successive configurations of the system on equally spaced times are stored for further analysis. In this way, microscopic configurations of the ring-shaped macromolecule are generated with the correct a priori probabilities.

The question we wish to answer now is which fraction of the stored configurations are knots and which fraction are topologically equivalent to a circle. In answering this question, one assumes that the random walk is self-avoiding; hence, when its shape is deformed, self-intersections are prohibited. The criterion for the existence of a knot is $\Delta(-1) \neq \pm 1$. The numerical algorithm consists of several stages. First, the configuration is projected onto a plane. Secondly, all trivial loops are removed in such a way that the number of double points decreases drastically. It turns out that this process reduces the number of double points considerable. For example, in a sample run with $N=320$, this process removed all double points for about $85 \%$ of all those configurations that turned out to be unknotted. Thirdly, for those configurations which cannot be reduced any further, the value of $\Delta(-1)$ is calculated with the standard method [3].

The fraction $\left(\zeta_{N}\right)$ of unknotted configurations of a closed macromolecule consisting of $N$ repeating units has been calculated for two geometries. In the first geometry, the molecule can move freely throughout all space. In the second geometry, the molecule is confined to the interior of a sphere of radius $R$. The re-
Table 1

The fraction $\zeta$ of unknotted rings as a function of the chain length $N l$ and the radius of the confining sphere, $R$. The total number of configurations studied in each case is denoted by $t$, of which $b$ were found to be unknotted rings.

\begin{tabular}{lllrl}
\hline & $R / l$ & \multicolumn{1}{l}{$l$} & \multicolumn{1}{l}{$b$} \\
\hline$N=64$ & $\infty$ & 25500 & 21548 & 0.845 \\
& 4 & 17000 & 12247 & 0.720 \\
& 3 & 16500 & 8972 & 0.544 \\
& 2.5 & 16500 & 6239 & 0.378 \\
& 2.1 & 19500 & 4207 & 0.216 \\
& 1.9 & 14400 & 2014 & 0.140 \\
$N=128$ & $\infty$ & 23500 & 15863 & 0.675 \\
& 5 & 16500 & 6890 & 0.418 \\
& 4 & 18100 & 4629 & 0.256 \\
$N=192$ & $\infty .5$ & 14400 & 2213 & 0.154 \\
& 8 & 38500 & 20789 & 0.540 \\
& 6.3 & 21900 & 8782 & 0.401 \\
& 5.5 & 18000 & 5099 & 0.283 \\
& 5 & 16800 & 3443 & 0.205 \\
$N=256$ & $\infty$ & 27420 & 15869 & 0.424 \\
$N=320$ & $\infty$ & 44220 & 15156 & 0.343
\end{tabular}

sults are presented in table 1 . Here, $R$ is measured in units $l$. Of the total number of configurations $(t)$ the number of unknotted configurations equals $b$; obvi-

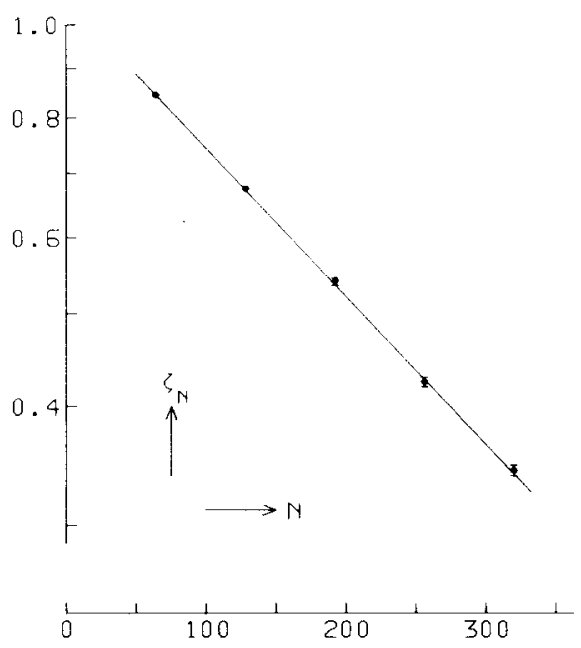

Fig. 1. Semi-logarithmic plot of the fraction $\zeta_{N}$ of unknotted rings as a function of the number of repeating units $N$. The uncertainty indicated by the error bars corresponds to $\pm 2 \sigma_{\mathrm{d}}$ where $\sigma_{\mathrm{d}}$ denotes the standard deviation $\left[\left(1-\xi_{N}\right) \xi_{N} / t\right]^{1 / 2}$. 
ously $\zeta_{N}=b / t$. These results are also plotted in figs. 1 and 2.

Before we turn to a discussion of these results, the following remark is appropriate. Due to the finite strength of the harmonic force between two neighbouring mass points, their distance $r_{i, i+1}$ is not exactly constant but fluctuates between narrow limits. For example, the probability that $\left|r_{i, i+1} / l-i\right|>0.02$ was smaller than $2 \%$.

When the results in fig. 1 and table 1 with $R / l=\infty$ are represented by a power law of the form

$\zeta_{N}=C \mu^{N} N^{\alpha}$

the best fit is found to be

$\mu=0.99646 \pm 0.00005, \quad|\alpha|<0.01$,

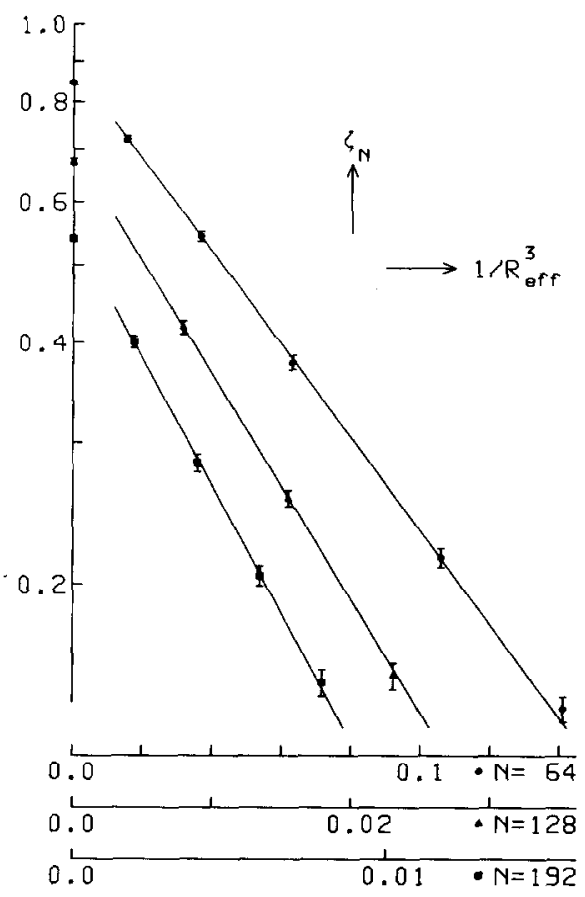

Fig. 2. Semi-logarithmic plot of $\zeta_{N}$ as a function of $R_{\text {eff }}^{-3}$ for three different values of $N$. Error bars as in fig. 1 . Note that the wall of the spliere to the interior of which the macromolecule is confined, is represented by a harmonic force which acts as soon as the distance to the center of the sphere is larger than $R$. Because of the finiteness of this force the effective radius $R_{\text {eff }}$ of the sphere is slightly larger than $R:$ in all cases but one we find $R_{\mathrm{eff}}=R+0.015 l$. For the case $R / l$ $=1.9$ we find $R_{\text {eff }}=R+0.02 l$. In this case the radius of the sphere is comparable to the length of a repeating unit. where the uncertainty indicated is given by \pm twice the standard deviation. Assuming $\alpha=0$, we find

$C=1.06 \pm 0.01$.

Thanks to the large number of configurations analyzed ( 44220 at $N=320$ to be compared with 173 at $N$ $=270$ in the work of des Cloizeaux and Mehta [5]), we were able to determine the value of the "topological exponent" $\alpha$ with fair numerical accuracy.

It is remarkable that $\alpha$ turns out to be so close to zero. The smallness of $\alpha$ suggests that this topological exponent has no relation to critical exponents. This is in contrast with the exponents which characterize self-avoiding random walks, which are indeed known to be related to critical exponents [31-33]. Hence, it may be conjectured that the topology of closed macromolecules is unrelated to the problem of counting self-avoiding random walks in a three-dimensional space. In this respect the two-dimensional case is different, cf. ref. [34].

The results in fig. 2 and table 1 for various values of $R / l$ and large $N$, can be represented by a scaling formula of the form

$\zeta_{N}(R) \sim \exp \left[-A\left(N^{\beta} l / R\right)^{\gamma}\right]$.

The best fit is found to be

$\gamma=3, \quad \beta=0.76$.

The scaling formula (4) is reminiscent of similar formulae in polymer physics [35]. At the time of writing, we can offer no explanation for the occurrence of scaling laws in these topological problems.

\section{References}

[1] M. Delbrück, in: Mathematical problems in the biological sciences, Proc. Symp. Appl. Math. 14 (1962) 55 (Americal Mathematical Society, Providence).

[2] G.M. Crippen, J. Theor. Biol. 45 (1974) 327.

[3] A.V. Vologodskii, A.V. Lukashin, M.D. FrankKamenetskii, and V.V. Anshelevich, Zh. Eksp. Teor. Fiz. 66 (1974) 2153 [Sov. Phys. JETP 39 (1974) 1059].

[4] M.D. Frank-Kamenetskii, A.V. Lukashin and A.V. Vologodskii, Nature 258 (1975) 398.

[5] J. des Cloizeaux and M.L. Mehta, J. de Phys. 40 (1979) 665.

[6] F.B. Fuller, Proc. Natl. Acad. Sci. USA 68 (1971) 815.

[7] F.B. Fuller, Proc. Natl. Acad. Sci. USA 75 (1978) 3557.

[8] F.H.C. Crick, Proc. Natl. Acad. Sci. USA 73 (1976) 2639.

[9] W.F. Pohl and G.W. Roberts, J. Math. Biol. 6 (1978) 383. 
[10] C.J. Benham, J. Mol. Biol. 123 (1978) 361.

[11] M. LeBret, Biopol. 19 (1980) 619.

[12] Y.D. Chen, J. Chem. Phys. 74 (1981) 2034.

[13] Y.D. Chen, J. Chem. Phys. 75 (1981) 2447.

[14] Y.D. Chen, J. Chem. Phys. 75 (1981) 5160.

[15] S. Prager and H.L. Frisch, J. Chem. Phys. 46 (1967) 1475.

[16] S.F. Edwards, Proc. Phys. Soc. 91 (1967) 513.

[17] R. Alexander-Katz and S.F. Edwards, J. Phys. A5 (1972) 674.

[18] N. Saito and Y.D. Chen, J. Chem. Phys. 59 (1973) 3701.

[19] F.W. Wiegel, J. Chem. Phys. 67 (1977) 469.

[20] F.W. Wiegel, Springer Lecture Notes Phys. 121 (1980) 78.

[21] A. Inomato and V.A. Singh, J. Math. Phys. 19 (1978) 2318.

[22] V. Tanikella and A. Inomata, Phys. Lett. 87 A (1982) 196.
[23] A.V. Vologodskii, V.A. Lukashin and M.D. FrankKamenetskii, Zh. Eksp. Teor. Fiz. 67 (1974) 1875 [Sov. Phys. JETP 40 (1975) 932].

[24] C.C. Gerry and V.A. Singh, Phys. Rev. D20 (1979) 2550.

[25] L.S. Schulman, J. Math. Phys. 12 (1971) 304.

[26] C. Bernido and A. Inomata, Phys. Lett. 77 A (1980) 394.

[27] F.W. Wiegel, Physica 109A (1981) 609.

[28] L. Verlet, Phys. Rev. 159 (1967) 98.

[29] J.P.J. Michels, Physica 90A (1978) 179.

[30] A.J. Kox, J.P.J. Michels and F.W. Wiegel, Nature 287 (1980) 317.

[31] P.G. de Gennes, Phys. Lett. 38A (1972) 339.

[32] J. des Cloizeaux, Phys. Rev. A10 (1974) 1665.

[33] J. des Cloizeaux, J. de Phys. 36 (1975) 281.

[34] F.W. Wiegel, Physica 98A (1979) 345.

[35] P.G. de Gennes, Scaling concepts in polymer physics (Cornell Univ. Press, Ithaca 1979). 